\title{
Thermal Modelling of Electrical Insulation System in Power Transformers
}

\author{
Agustín Santisteban, Fernando Delgado, \\ Alfredo Ortiz, Carlos J. Renedo and Felix Ortiz \\ Additional information is available at the end of the chapter
}

http://dx.doi.org/10.5772/intechopen.78070

\begin{abstract}
Temperature is one of the limiting factors in the application of power transformers. According to IEC 60076-7 standard, a temperature increase of $6^{\circ} \mathrm{C}$ doubles the insulation ageing rate, reducing the expected lifetime of the device. Power losses of the transformer behave as a heating source, and the insulating liquids act as a coolant circulating through the windings and dissipating heat. For these reasons, thermal modelling becomes an important fact of transformer design, and both manufacturers and utilities consider it. Different techniques for thermal modelling have been developed and used for determining the hot-spot temperature, which is the highest temperature in the winding, and it is related with the degradation rate of the solid insulation. First models were developed as a first estimation for modelling the hot-spot temperature and the top-oil temperature. These models were based on thermal-electric analogy and are known as dynamic models. Other two different kinds of models are widely used for thermal modelling, known as Computational Fluid Dynamics (CFD) and Thermal Hydraulic Network Models (THNMs). These two techniques determine the temperature and velocity fields in the winding and in the insulating fluid. In this chapter, the different techniques for transformer thermal modelling will be introduced and described.
\end{abstract}

Keywords: thermal modelling, power transformer, electrical insulation system, CFD, THNM

\section{Introduction}

Power transformers are key devices in the electrical grids, and this is a main reason for utilities and manufacturers to improve its performance and lifetime expectancy. Although its performance 


\begin{tabular}{lll}
\hline$\theta_{\mathbf{h}}\left({ }^{\circ} \mathbf{C}\right)$ & Normal paper & Thermally upgraded paper \\
\hline 80 & 0.125 & 0.036 \\
86 & 0.25 & 0.073 \\
92 & 0.5 & 0.145 \\
98 & 1.0 & 0.282 \\
104 & 2.0 & 0.536 \\
110 & 4.0 & 1.0 \\
116 & 8.0 & 1.83 \\
122 & 16.0 & 3.29 \\
128 & 32.0 & 5.8 \\
134 & 64.0 & 10.1 \\
140 & 128.0 & 17.2 \\
\hline
\end{tabular}

Table 1. Relative ageing rate for different hot-spot temperatures [1].

is over $99 \%$, heat generation becomes a key factor for power limitation and expected lifetime. Transformer insulation ageing is sensible to temperature, doubling the ageing rate with a $6^{\circ} \mathrm{C}$ increase over the designed temperature according to IEC Standard 60076-7 [1]. High temperatures are caused by losses in the device, mainly due to Joule losses and eddy losses in the windings. The transformer insulation system that consists of a dielectric fluid and a solid insulation is the most critical part, and its degradation is related with the expected lifetime of the device [1]. The standard previously cited proposes a formulation to determine the ageing acceleration with the temperature

$$
\begin{gathered}
U=2^{\left(\left(\theta_{h}-98\right) / 6\right)} \\
U=e^{\left(\frac{15000}{110+273}-\frac{15000}{\theta_{h}+273}\right)}
\end{gathered}
$$

where $\mathrm{V}$ is the relative ageing rate of the insulation, which is calculated by Eq. (1) for normal insulation paper and by Eq. (2) for thermally upgraded insulation paper, and $\theta_{\mathrm{h}}$ refers to the hot-spot temperature $\left({ }^{\circ} \mathrm{C}\right)$. Both equations indicate that the relative ageing rate is sensible to hot-spot temperature variations as shown in Table 1.

For this reason, the estimation of the hot-spot temperature becomes an important task for manufacturers and utilities. Different models have been developed for the study of hot-spot temperature in oil-immersed power transformers that will be introduced in the following sections.

\section{Dynamic models}

The first thermal modelling technique described is the dynamic thermal model. These models are based on the thermoelectric analogy to design a circuit based on the thermal resistance and 
thermal capacitance of the transformer [2]. The circuit is modelled by a partial differential equation whose solution gives the evolution of the hot-spot temperature over time. Normally, to simplify the solution, an intermediate circuit often comes with the hot-spot temperature model to obtain the top-oil temperature, Figure 1.

These models are associated with the differential equations, Eqs. (3) and (4) [2].

$$
\begin{gathered}
\frac{1+R \cdot K^{2}}{1+R} \cdot \mu_{p u}^{n} \cdot \Delta \theta_{\text {oil, rated }}=\mu_{p u}^{n} \cdot \tau_{\text {oil, rated }} \cdot \frac{\partial \theta_{\text {oil }}}{\partial t}+\frac{\left(\theta_{\text {oil }}-\theta_{\text {amb }}\right)^{1+n}}{\Delta \theta_{\text {oil, rated }}^{n}} \\
\left\{K \cdot P_{c u, p u}\left(\theta_{h s}\right)\right\} u_{p u}^{n} \cdot \Delta \theta_{\mathrm{hs}, \text { rated }}=\mu_{p u}^{n} \cdot \tau_{\mathrm{wdg}, \text { rated }} \cdot \frac{\partial \theta_{h s}}{\partial t}+\frac{\left(\theta_{h s}-\theta_{\text {oil }}\right)^{n+1}}{\Delta \theta_{\mathrm{hs}, \text { rated }}^{n}}
\end{gathered}
$$

where the terms are as follows: $\mathrm{R}$, ratio of rated load losses to no-load losses; $\mathrm{K}$, the load factor; $\mu_{\mathrm{pu}}$, oil viscosity (per-unit value); $\theta_{\mathrm{amb}}$, ambient temperature $\left({ }^{\circ} \mathrm{C}\right.$ ); $\theta_{\mathrm{hs}}$, hot-spot temperature $\left({ }^{\circ} \mathrm{C}\right) ; \theta_{\text {oil, }}$ top-oil temperature $\left({ }^{\circ} \mathrm{C}\right) ; \Delta \theta_{\text {oil,rated, }}$ rated top-oil temperature rise over ambient ( $\left.{ }^{\circ} \mathrm{C}\right) ; \Delta \theta_{\text {hs,rated, }}$ rated hot-spot temperature rise over top-oil temperature $\left({ }^{\circ} \mathrm{C}\right)$;

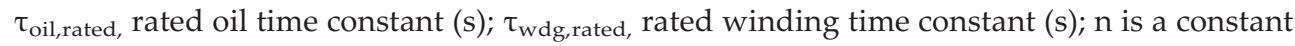
obtained from tables; and $\mathrm{P}_{\mathrm{cu}, \mathrm{pu}}(\theta \mathrm{hs})$ load loss's dependence on temperature (per-unit value).

In addition, the IEC 60076-7 standard proposes their own method with Eqs. (5) and (6):

$$
\begin{gathered}
\theta_{h}(t)=\theta_{a}+\Delta \theta_{o i}+\left\{\Delta \theta_{o r} \cdot\left[\frac{1+R \cdot K^{2}}{1+R}\right]^{x}-\Delta \theta_{o i}\right\} \cdot f_{1}(t)+\Delta \theta_{h i}+\left(H \cdot g_{r} \cdot K^{y}-\Delta \theta_{h i}\right) \\
\theta_{h}(t)=\theta_{a}+\Delta \theta_{o r} \cdot\left[\frac{1+R \cdot K^{2}}{1+R}\right]^{x}+\left\{\Delta \theta_{o i}-\Delta \theta_{o r} \cdot\left[\frac{1+R \cdot K^{2}}{1+R}\right]^{x}\right\} \cdot f_{3}(t)+H \cdot g_{r} \cdot K^{y}
\end{gathered}
$$

where Eq. (5) is applied for increasing the load factor and Eq. (6) is applied for decreasing the load factor. The functions $\mathrm{f}_{1}, \mathrm{f}_{2}$ and $\mathrm{f}_{3}$ can be calculated as explained in [1]. With these two models, the hot-spot temperature of a power transformer can be predicted over time, under different load conditions. In [2], both methods are tested with different transformers of different power rates and contrasted its accuracy with experimental measurements.

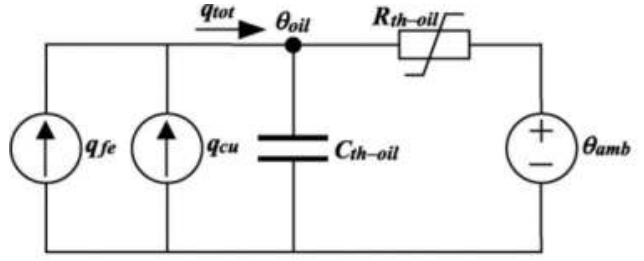

(a)

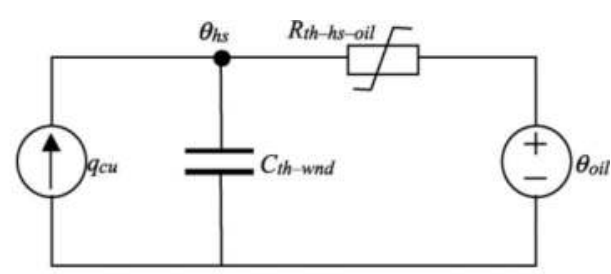

(b)

Figure 1. Dynamic models presented in [2]. (a) Top-oil model and (b) hot-spot model. 


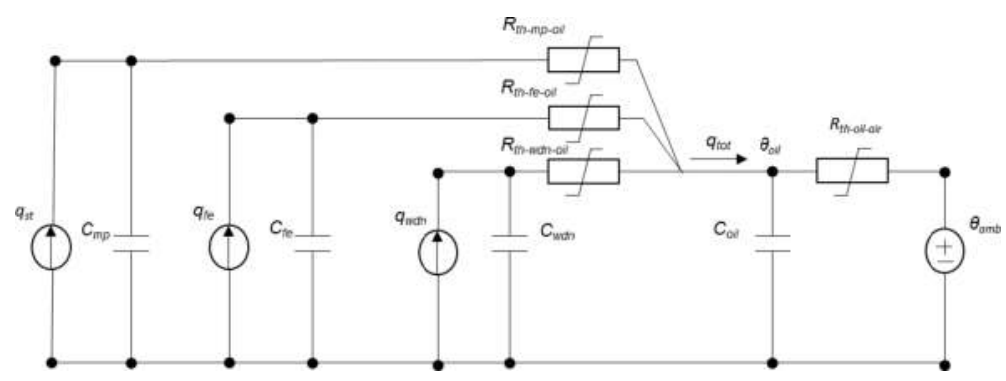

Figure 2. Complex top-oil thermal circuit [3].

For both models, a large number of parameters obtained from heat-run tests are necessary for calculations. However, the model proposed in [2] requires less input data than the model proposed by the standards.

Other complex models have been developed for top-oil and hot-spot temperature prediction. These models take into account the different thermal resistances that appear from the different heating sources in power transformer as shown in Figure 2 [3]. In addition, models for bottomoil temperature and bottom-winding temperature have been developed in [4].

In conclusion, dynamic thermal models are useful and can be implemented in computer software without hardly computational cost, requiring less than $5 \mathrm{~min}$ in a normal PC. However, the input data necessary to run the model come from the results of heat-run tests, which made this method dependent on the knowledge of these results.

\section{Steady-state models}

An alternative to dynamic models is steady-state model. Steady-state models attempt to predict the temperature field in the transformer winding, determining the hot-spot temperature and its location. In these modelling techniques, the geometrical parameters of the transformer winding gain importance. Among the former, the one that is based on heat-run test is shown in the next section. Then, between the second ones, models based on Computational Fluid Dynamics (CFD) and those based on Thermal-Hydraulic Network Modelling (THNM) are developed.

\subsection{Hot-spot temperature rise from normal heat-run test data}

The fundamental objective of a transformer thermal modelling is to be able to accurately predict winding and component temperature rises above ambient temperature. For the industry and transformer users, the determination of the location of the hot-spot and the hot-spot temperature rise is especially interesting. In [1], the limit temperature for top oil and hot spot is established at 105 and $120^{\circ} \mathrm{C}$ for normal operation. 
These temperature rises are caused by the combination of voltage-related losses (no-load losses) and current-related losses (load losses). The loss distribution is a scalar function $\mathrm{P}(\mathrm{r}, \varphi$, $\mathrm{z})\left[\mathrm{W} / \mathrm{m}^{3}\right]$ over the volume of these transformer metal parts. The determination of electromagnetic (EM) losses is usually made using the Finite Element Method (FEM). Windings are divided into many rectangular sections with a uniform ampere-turn distribution. The eddy losses are calculated for each conductor using Eq. (7)

$$
P=\frac{\omega^{2} \cdot B^{2} \cdot T^{2}}{24 \cdot \rho}
$$

where $B$ is the peak leakage flux density $(T), \omega=2 \pi f$, where $f$ is the frequency $(\mathrm{Hz}), T$ is the conductor dimension perpendicular to the direction of the leakage flux density $(\mathrm{m})$ and $\rho$ is the resistivity $\left(\Omega \cdot \mathrm{m} / \mathrm{mm}^{2}\right)$.

The axial and radial flux densities are assumed to be constant over a single conductor and equal to the value at its centre. The total eddy losses for each winding are calculated by integrating the losses of all its conductors.

The boundary conditions can influence the results. A transformer is a 3D construction, in which the windings usually are symmetric, but the surrounding steel parts (core and tank) are not, Figure 3.

The temperature rise over ambient temperature has to be calculated based on this loss distribution taken into account thermal conductivity of the metal, the thermal conductivity of the electrical insulation and convective cooling due to oil flow (viscosity of oil is important, which makes the oil temperature also an important parameter).

The temperature rise of a transformer winding above the ambient temperature is built up from three component temperature rises: (1) the temperature rise of the inlet oil, (2) the temperature rise of the cooling oil as it passes through the transformer and (3) the temperature rise of the

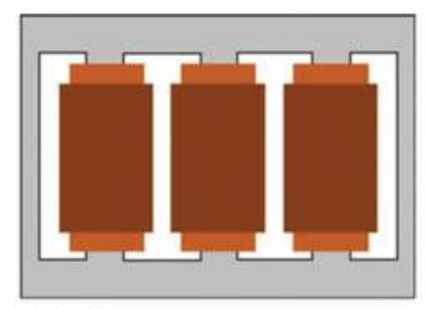

Front view

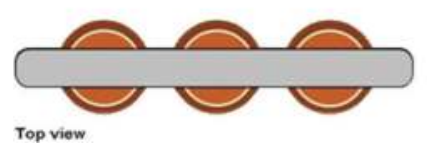

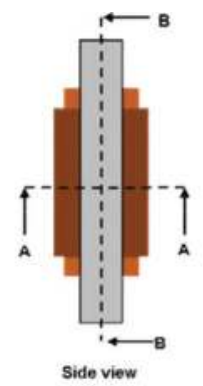

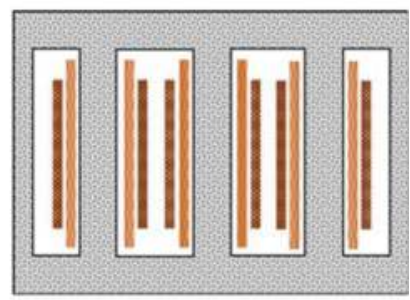

Cross section $B$

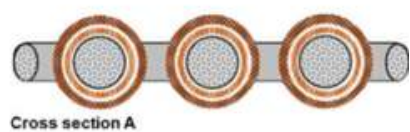

Figure 3. Three-phase transformer with five-legged core and LV winding with a large pitch (front, top and side views, and cross sections). 
winding above the cooling oil. These temperatures can be measured in the surrounding of the transformer and in three points of it: inlet and outlet oil temperatures and mean winding temperature.

The temperature increase of the oil on passing through the transformer is the difference between the inlet and the outlet oil temperatures, but in a transformer, usually there are more than one parallel oil paths, and each one could have their own temperature rise. It is accepted that this increase is approximately the difference between the top-oil temperature and the cooled oil inlet temperature [1].

The temperature rise of the winding above the oil is referred to as the "gradient," g. A mean value for this is obtained from the difference between the mean winding temperature, usually determined by resistance measurements, and the mean oil temperature. The gradient can vary with position because of local variations in winding losses and cooling effectiveness.

The temperature rise at the hot spot, $\Delta \theta_{\mathrm{h}}\left({ }^{\circ} \mathrm{C}\right)$, top-oil temperature, $\Delta \theta_{\mathrm{o}}\left({ }^{\circ} \mathrm{C}\right)$, is expected to be greater than the mean winding gradient, g, as expressed by a hot-spot factor, H, Eq. (8):

$$
\Delta \theta_{h}=\Delta \theta_{0}+(H \cdot g)
$$

where $\mathrm{H}$ could be expressed as the product of two dimensionless factors, $\mathrm{Q}_{\mathrm{fac}}$, related to additional loss, and $\mathrm{S}_{\mathrm{fac}}$ related to the efficiency of cooling, Eq. (9):

$$
H=Q_{f a c} \cdot S_{f a c}
$$

The $\mathrm{H}$ factor can be derived from Figure 4 and can be expressed according to Eq. (10):

$$
H=\frac{\theta_{h}-\theta_{0}}{\theta_{w}-\frac{\theta_{0}-\theta_{b}}{2}}
$$

where $\theta_{\mathrm{w}}$ and $\theta_{\mathrm{b}}$ are, respectively, the average winding and bottom-oil temperatures $\left({ }^{\circ} \mathrm{C}\right)$.

The Q-factor is a dimensionless factor as a ratio of two losses, and in cylindrical coordinates is defined according to Eq. (11)

$$
Q_{f a c}=\frac{q(r, z, \varphi, \mathrm{T})}{q_{a v e}}
$$

where $q(r, z, \varphi, T)$ is the local loss density at a location $\left(W / m^{3}\right), r$ is the radial position, $\varphi$ is the angle in circumferential position, $\mathrm{z}$ is the axial position, $\mathrm{T}$ is the local temperature at $(\mathrm{r}, \mathrm{z}, \varphi)(\mathrm{K})$ and $\mathrm{q}_{\mathrm{ave}}$ is the average loss of the winding at an average temperature $\left(\mathrm{W} / \mathrm{m}^{3}\right)$.

Heat transfer can be propagated in different directions, the overall heat transfer being a series and parallel parts of (1) the insulation between the neighbouring conductors that are in direct contact with each other. This is in a radial direction. (2) The insulation paper and oil boundary layer between the conductor and the oil flow. This is in an axial direction. (3) The winding copper. This is located in the tangential direction and usually can be neglected. 


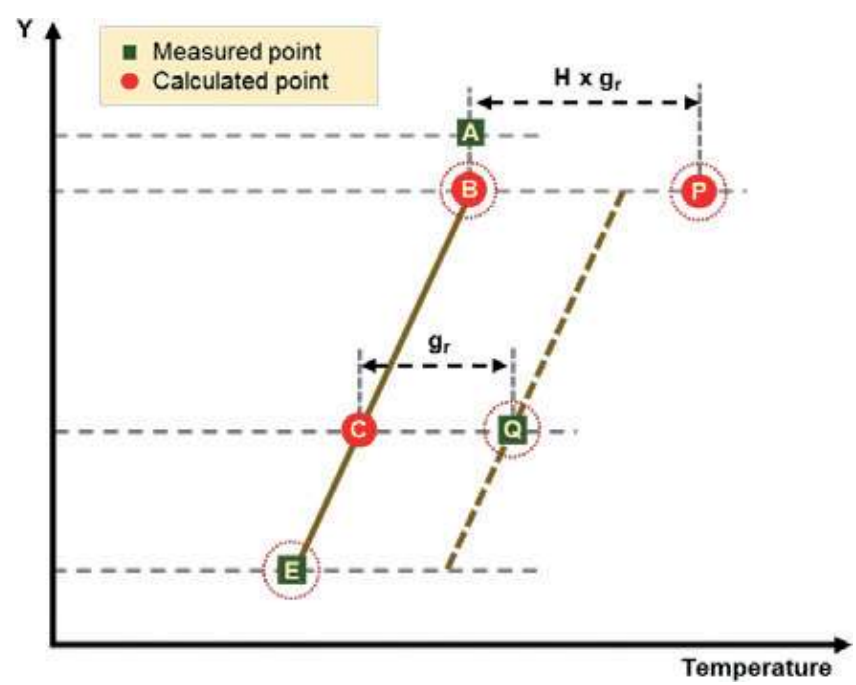

Figure 4. Transformer thermal diagram with the measured quantities highlighted. A-Top-oil temperature derived as the average of the tank outlet oil temperature and the tank oil pocket temperature. B-Mixed oil temperature in the tank at the top of the winding (often assumed to be the same temperature as A). C-Temperature of the average oil in the tank. $\mathrm{D}$-Oil temperature at the bottom of the winding. E-Bottom of the tank. gr-Average winding to average oil (in tank) temperature gradient at rated current. $\mathrm{H}-$ Hot-spot factor. $\mathrm{P}-$ Hot-spot temperature. $\mathrm{Q}-$ Average winding temperature determined by resistance measurement.

For transformer manufacturers, a good thermal design is a very important issue. Aspects as material, winding geometry, oil paths through the windings, or oil velocity are very relevant. This design determines the cost and the insulation ageing (transformer life).

Mathematical models allow predicting transformer temperatures and winding hot-spot temperatures with good accuracy. Transformer manufacturers use these models to make their designs and any significant design variants. However, simulating the loss distribution in the structural metal parts of a transformer requires a very large number of small mesh elements. Nevertheless, calculating the temperature rise distribution in the structural metal parts requires a much higher resolution mesh density.

\subsection{Computational fluid dynamics modelling}

Tackling numerically, a complex physical problem using computational resources has only been able since few years ago. In fact, the current powerful computing resources allow us to discretize complex geometries. Then, the underlying governing equations, for example, differential equations (DEs), can be solved assuming small simplifications. The discretization process converts these DEs in a set of algebraic equations that can be solved using numerical algorithms. Regarding our topic, in which heat transfer and fluid dynamics physics are involved, the numerical method that carries out this process is named Computational Fluid Dynamics (CFD). 


\subsubsection{CFD basic concepts}

CFD is based on the solution of the Navier-Stokes equations. These are a set of Partial Differential Equations (PDE) that state the mass, momentum and energy conservations, Eqs. (15)(17). However, it is not possible to solve them in an exact way (except some scarce and special cases). CFD allows solving these PDE by means of the geometrical model discretization. The latter converts the PDE in a set of algebraic equations that can be solved using specialised numerical algorithms, such as Gauss elimination algorithm, on a computer. These algorithms obtain the solution (velocity, pressure, temperature, in the case of power transformers) at discrete points of the studied domain [5]

$$
\begin{gathered}
\nabla \cdot(\rho u)=0 \\
\rho(u \cdot \nabla) \mathrm{u}=\nabla\left[p I+\mu\left(\nabla u+(\nabla u)^{T}\right)-\frac{2}{3} \mu(\nabla \cdot u) I\right]+F \\
\rho C_{p} u \cdot \nabla T=\nabla \cdot(k \nabla T)+\mathrm{q}
\end{gathered}
$$

where $\rho, u, p, \mathbf{I}, \mu, \mathrm{F}, \mathrm{C}_{\mathrm{p}} \mathrm{T}$ and $q$ of Eqs. (12)-(14) are, respectively, density $\left(\mathrm{kg} / \mathrm{m}^{3}\right)$, velocity vector $(\mathrm{m} / \mathrm{s})$, pressure $(\mathrm{Pa})$, identity matrix, dynamic viscosity $(\mathrm{Pa} \cdot \mathrm{s})$, body force vector $\left(\mathrm{N} / \mathrm{m}^{3}\right)$, specific heat capacity $(\mathrm{J} / \mathrm{kg} \cdot \mathrm{K})$, temperature $(\mathrm{K})$ and unitary heat transfer $\left(\mathrm{W} / \mathrm{m}^{3}\right)$.

The first step in this kind of modelling is usually to determine the dimensions number of the geometrical model: $2 \mathrm{D}$ or $3 \mathrm{D}$, generally. This is very important since the geometrical model is the first source of errors. For instance, if 2D models were considered in CFD, important edge effects that contribute to heat transfer and flow phenomena could be obviated.

Generally, CAD tools are used to draw the geometry (Solidworks, Inventor, etc.). These tools allow defining the geometry with a high-detail level. The latter is an essential aspect to be considered since a very detailed geometry can excessively complicate the numerical model without significant improvement in the problem definition. For instance, in the case of the power transformer shown in Figure 5, it was decided to simplify the phase using angular and plane symmetries since these simplifications did not affect the solution accuracy.

The physics involved in the problem analysed must be described. In CFD, many mathematical models can be used to define the heat transfer and flow phenomena. In fact, an adequate selection of the model and a correct setting of the boundary conditions supported by this model are a crucial task to avoid errors in the numerical solution obtained.

This task consists in the division of the geometric model in smaller parts (cells). This is the socalled discretization. There are mainly three discretization techniques: finite element, finite volume and finite difference. At the same time, these techniques generate structured and/or unstructured meshes.

Structured meshing is habitually used in simple geometries such as the one shown in Figure 5. This geometry consists in volumetric cells of six faces that can uniquely be identified using 

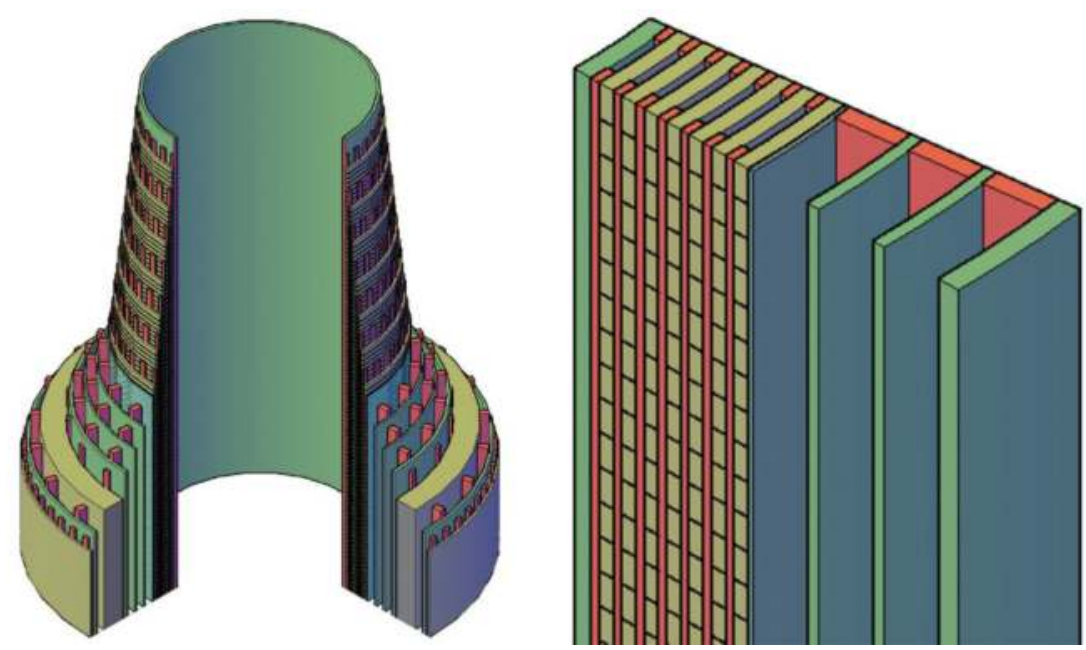

Figure 5. Geometry definition.

three indexes $(\mathrm{i}, \mathrm{j}, \mathrm{k})$. The deformation grade (mesh quality) of the elements of this meshing type is generally smaller than in the other case. In addition, the cells can be oriented in the main flow direction, thus capturing the flow phenomena in a better way.

By contrast, unstructured meshing can be used in very complex geometries. The cells may have any shape (quadrilateral or triangular shapes in 2D, tetrahedral or hexahedral shapes in $3 \mathrm{D})$, thus a better adaption to the geometry is obtained. However, this type of mesh generates a set of algebraic equations whose solution time is habitually higher. Finally, the mesh refinement grade affects the solution accuracy. Generally, the finer the mesh, the more accurate the solution is.

The solution of the set of algebraic equations resulting from the discretization of the geometric model is the last step. This can be done using direct or iterative methods. The latter are habitually used to solve the Conjugate Heat Transfer (CHT) problems since the computational requirements are smaller than that required by the former methods. For instance, CHT problems are commonly solved using iterative methods such as Conjugate gradient, Gauss-Seidel and Multigrid.

In addition, to solve the algebraic equations, a convergence criterion is needed to be considered. This is often implied to assume a maximum error in the residuals of the governing equations. That is, the solution obtained is not exact. In other words, another error appears in the numerical model solution.

In comparison with other numerical tools (for instance, THNM), a lot of computing time and a great amount of computational resources are needed by CFD. However, this technique allows knowing the fluid flow and heat transfer phenomena in fine detail. 


\subsubsection{Main research lines of CFD applied to transformer thermal modelling}

As can be seen in Figure 6, the cooling system of a power transformer is a closed loop that consists mainly of a heat source (windings), a heat sink (radiators) and a tank. Most CFD studies are focused on the thermal-fluid behaviour of the cooling system inside the windings. Nonetheless, some efforts have been put into the thermal modelling of the radiators too. The next subsections present a brief review of these topics.

\subsubsection{Winding modelling}

As mentioned previously, the main goal of the winding CFD modelling is to numerically predict the hot-spot temperature and temperature distributions in oil-immersed transformers since their life span depends on it. First works were developed at the beginning of this century. For instance, Mufuta et al. and El Wakil et al. modelled two windings using this technique. The former characterises the oil flow through an array of discs with different spaces between discs and different inlet conditions [7]. The latter employed a 2D axisymmetric model of a power transformer with six different geometries and six different inlet velocities in order to study the heat transfer and oil flow through the windings [8]. In the same decade, other authors have contributed to this labour. For instance, Torriano performed 2D and 3D simulations of an LV winding (LVW) of a power transformer with zigzag cooling to determine the effects of several elements, such as sticks and inter-sticks, in the temperature distribution [6, 9]. In 2011, Gastelurrutia et al. carried out a study where they developed a 3D and a 2D model of an Oil Natural-Air Natural (ONAN) distribution transformer. They demonstrated the good capacity of the simplified 2D model to represent the thermal behaviour of the whole transformer [10]. In 2012, Tsili et al. established a methodology to develop a 3D model to predict hot-spot temperature [11]. In this year, Skillen et al. carried out a CFD simulation of a 2D non-isothermal flow axisymmetric model in order to characterise the oil flow in transformer winding with zigzag

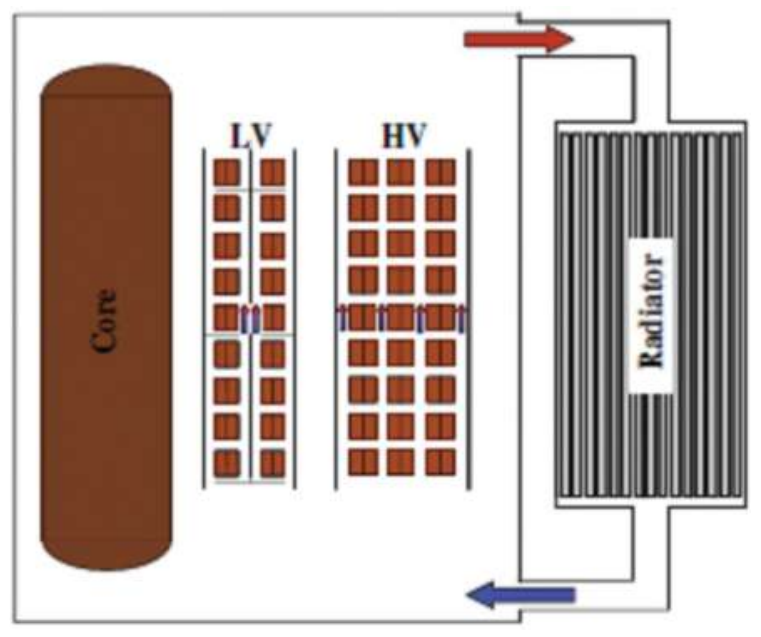

Figure 6. Schematic of oil circulation in a power transformer [6]. 
cooling [12]. In 2014, Yatsevsky carried out a 2D-axisymmetric simulation of a CHT model of a transformer, including the core, the tank and the radiator, in order to predict hot spots in an oil-immersed transformer with natural convection. The developed model has shown a good adequacy verified by experiments [13]. Recently, Torriano et al. have developed a 3D CHT model of an Oil Natural (ON) disc-type power transformer-winding scale model. An underestimation of the average and hot-spot temperatures was obtained in this model in comparison with the experimental setup when the entire cooling loop was considered. This is the reason why the authors chose to reduce the computational domain to the winding, setting the inlet boundary conditions. This way, the model accuracy was improved significantly [14].

The substitution of mineral oil by new biodegradable dielectric liquids is another research line in which CFD is used as an analysis tool. However, few experimental and theoretical works can be found in the study related with the cooling capacity of these new liquids. In 2015, Park et al. employed a 2D-CFD model to obtain temperature and velocity profiles of some alternative liquids used in a distribution transformer of 2.3 MVA and a power transformer of 16.5 MVA [15]. In the same year, Lecuna et al. carried out a 3D-CFD simulation of an ONAN distribution transformer comparing a natural ester, a synthetic ester, a high kinematic viscosity silicone oil and a low kinematic viscosity silicone oil with a mineral oil [16]. These works conclude that alternative liquids produce higher temperatures in the transformer windings designed for mineral oil. More recently, Santisteban et al. evaluated the cooling performance of two alternative vegetal liquids with that of a typical mineral oil. This task was carried out using a 2D-axisymmetric model of an LVW with zigzag cooling in which temperature distributions, hot-spot temperatures and their locations, and hot-spot factors were determined. In contrast to the results of the previous works, this work shows that the hot-spot temperature is lower for the vegetable oils in the initial design than that of mineral oil [17].

Finally, CFD is also used to analyse the advantage of using natural esters in the transformer insulation system. For instance, in 2016, Fernandez et al. published a work in which laboratory experiments and CFD simulations are combined to study the influence of vegetable oils in the life span of the winding insulation paper [18]. It was concluded that, even though the chapter suffers worse thermal conditions when it is immersed in vegetable oils, the physical properties of these oils extend the life span of this chapter, Figure 7. That is, in the long term, both effects tend to the balance and the degradation is similar to the one obtained in windings cooled by mineral oil.

\subsubsection{Radiator modelling}

Most of the power transformers have fan-cooled radiators. CFD can be used to improve the cooling capability of these components. Few works can be found with this subject. In fact, this topic has mainly begun to be treated in this decade. For instance, Kim et al. presented in 2013, a predictive and experimental study about the cooling performance of the radiators used in oil-filled power transformers with two different cooling methods, ONAN and Oil-Directed Air Natural (ODAN) [19]. The aim was to experimentally evaluate the cooling capacity of the radiator and compare the results with those obtained with two different predictive methods. CFD was one of these methods. The authors stated that the radiator optimization could be 

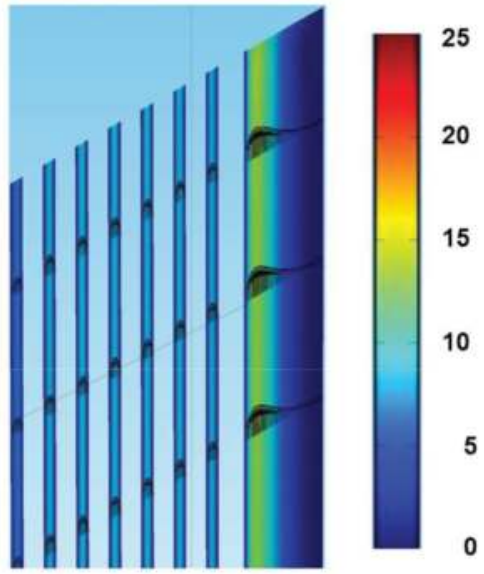

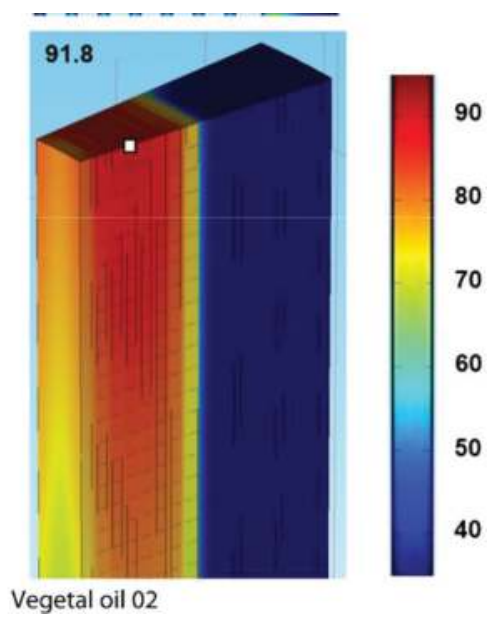

Figure 7. Velocities $(\mathrm{mm} / \mathrm{s})$ and temperatures $\left({ }^{\circ} \mathrm{C}\right)$ distributions.

done in this way. On the other hand, to improve the cooling capacity of the radiators, the fan's location in the radiators has to be studied. Paramane et al. conducted this in 2014 using both CFD and experimental studies [20]. They considered horizontal and vertical blowing directions. For the transformer studied, they found that the horizontal blowing direction had a higher performance due to the lesser air sideway leakages that those of the vertical blowing case. Two years later, in 2016, the same authors carried out the same type of study [21]. However, as a novelty, they provide the effect of the blowing direction on the temperature and velocity distributions of the oil inside the radiators.

Finally, in 2017, Ríos et al.? presented the result comparison of two models (a semi-analytical model and a CFD model) with the experimental results of a radiator of a 30-MVA power transformer working in ONAN mode. The objective was to validate both models in order to use them in the optimization of the current radiator design [22]. This aim was accomplished. An extension of this work was presented the same year with the goal of analysing the thermal-fluid dynamic behaviour of the radiator working in ONAF mode with vertical blowing of the fans [23]. The results obtained, which were validated with experimental and CFD results, showed that the semi-analytical model they proposed was a useful tool for radiator design processes.

\subsection{Thermal hydraulic network modelling, THNM}

THNM is a technique for transformer thermal modelling that relies on three basic principles: mass conservation, momentum conservation and energy conservation. It implies a subdivision of the domain in multiple elements where the conservation principles are observed as a convergence condition.

This model describes the conservation principles by algebraic equation sets that makes the solving time shorter than CFD that describes the same principles into a set of partial differential 
equations. These principles are applied to solve the temperature and oil flow fields in several parts of the transformer (windings, core, coolers). THNM applied on the windings can be useful for predicting the hot-spot temperature.

THNM models are the coupling of two different networks: the hydraulic network and the thermal network. The hydraulic network comprises the oil flows through channels and nodes. The oil flow is modelled using the electric circuit analogy where the oil flow and pressure correspond to, respectively, electrical current and voltage. The thermal network models the heat transfer between the active parts and the cooling circuit of the transformer. Temperaturedependent properties of the materials and heat transfer coefficients make both networks to be coupled.

\subsubsection{Hydraulic network}

The hydraulic network corresponding to a disc winding with barriers is shown in Figure 8 . The hydraulic network consists of eight nodes, four per axial side, where two of the nodes correspond to the entrance and exit of the coolant and the three remaining nodes per side are where the coolant branches or merges. The network is represented by its electrical circuit analogy where the physical quantity associated to each node corresponds to the sum of the static and dynamic pressures. In the circuit, resistances represent frictional pressure drops, and resistances in the nodes represent the local pressure drops as well. Buoyancy effect is represented by generators representing the gain of pressure due to gravitational effects. Since the hydraulic resistances depend on the flow, the hydraulic circuit proposed in Figure 8 is a nonlinear circuit that has to be solved with an iterative procedure [24].
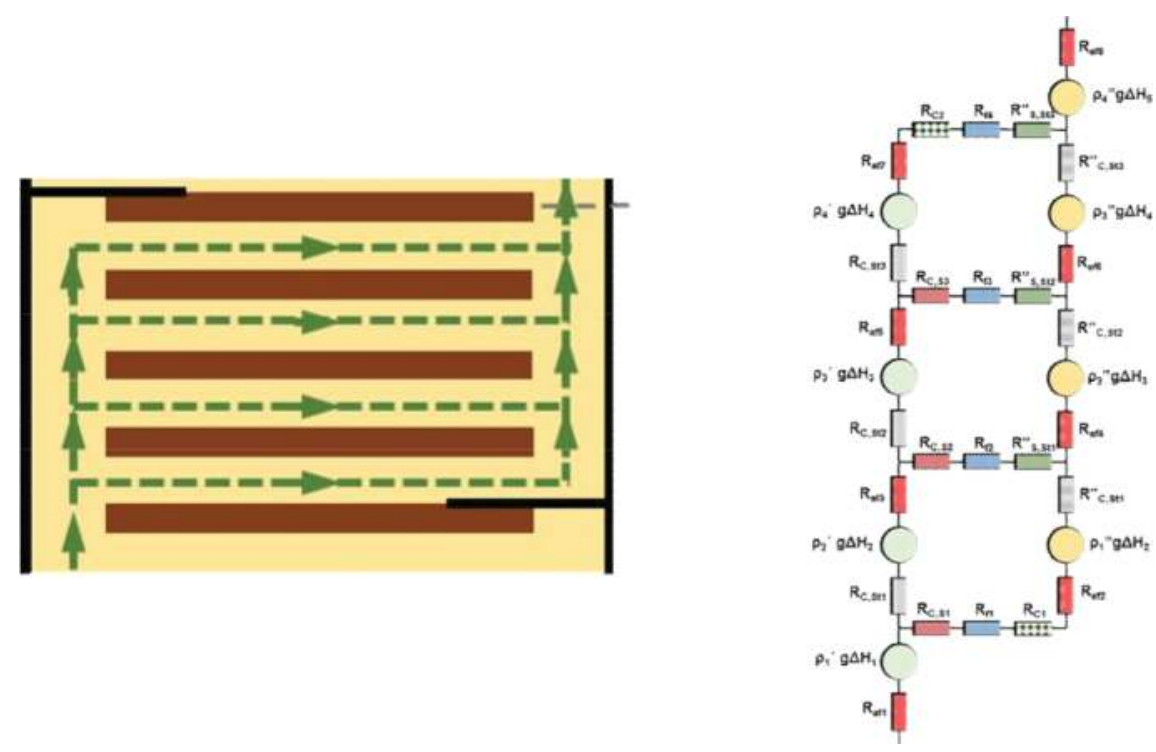

Figure 8. Analogy hydraulic circuit versus hydraulic network. 


\subsubsection{Thermal network}

Thermal network describes the heat transfer phenomena on the transformer and on the transformer elements. There are two main mechanisms of heat transfer in a transformer winding: heat conduction in the conductors and in the solid insulation, and convection from the active part to the cooling oil. The convection term highly depends on the oil flow distribution, which is given by the hydraulic network. In addition, hydraulic network depends on temperature buoyancy effect and temperature-dependent properties of the cooling oil. This coupling between networks enhances the necessity to apply iterative procedures to solve both networks.

An approach for the thermal part of a transformer winding is to build a thermal network. For the case of a disc winding, the analogy with electric circuits is useful to model the heat transfer in two directions, axial and radial. A resistive term is used for modelling heat conduction between conductor and solid insulation and a resistive term is used for the convective part, which depends on the oil flow distribution. A good approach consists in assuming that the thermal resistance of the conductor is negligible, considering only the resistance of the solid insulation. A voltage source represents the oil temperature and a current source represents the heat generation on each node. Figure 9 represents the thermal network previously described, where the $R_{\lambda i}$ represents the thermal resistance for conductive terms, $R_{\alpha i}$ represents the thermal resistance for convective terms, $\theta_{\mathrm{bi}}$ and $\theta_{\mathrm{ti}}$ represent the temperature on the channels and $\mathrm{P}_{\gamma \mathrm{i}}$ represents the heat source in the conductors [24].

\subsubsection{Complete loop modelling}

Other application of THNM is to model the complete oil loop of the transformer. It is based on the global pressure equilibrium of the oil loop, considering core, tank, winding and radiators, taking into account thermal driving forces, pump forces and pressure drops in the whole loop. Thermal driving forces appear due to density changes with temperature. Driving forces and pressure drop depend on the oil flow rate. The oil flow rate, $Q_{0}$, represents the equilibrium between driving forces and pressure drops.
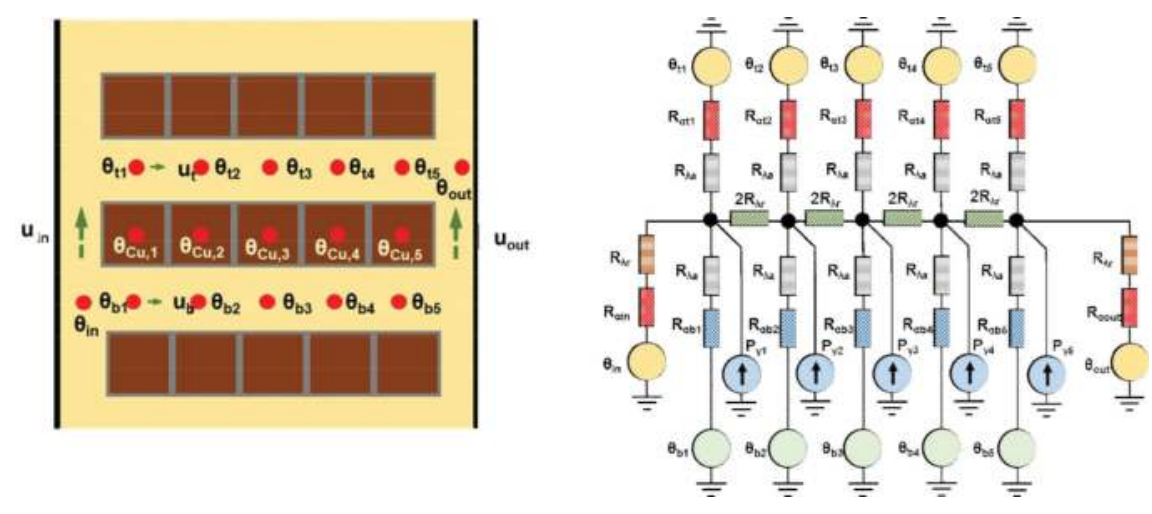

Figure 9. Thermal network of a transformer winding disc. 
A simple way to imagine the oil loop of a transformer is described as follows: the oil is heated in the windings, then flows through a piping system reaching the radiator, where it is cooled and finally goes through another piping system to reach the starting point. Although there is heat exchange in the piping system, it is considered negligible compared to the heat exchanged in radiators and windings. It is represented in Figure $\mathbf{1 0 .}$

Thermal driving force is generated due to density variations along the loop and can be expressed by Eq. (15)

$$
p_{T}=\oint \rho \cdot \vec{g} \cdot \overrightarrow{d l}=\oint \rho \cdot g \cos \varphi \cdot d l
$$

where $\rho$ is the oil density, $\vec{g}$ is the gravity vector, $\varphi$ is the angle between velocity and gravity vectors and $\vec{l}$ is the path vector.

For simplicity, Eq. (16) can be expressed as follows:

$$
p_{T}=\rho_{r} \cdot g \cdot \beta \cdot \Delta \theta_{o l} \cdot \Delta H
$$

where $\rho_{\mathrm{r}}$ is the oil density at a reference temperature $\left(\mathrm{kg} / \mathrm{m}^{3}\right), \beta$ is the volume expansion coefficient of the oil $(1 / \mathrm{K}), \Delta \theta_{\mathrm{Ol}}$ is the vertical temperature gradient $\left({ }^{\circ} \mathrm{C}\right)$ and $\Delta \mathrm{H}$ is the height difference between the centre point of the radiator and the centre point of the winding $(\mathrm{m})$.

When the pump runs directly to the windings, the total driving pressure will be the sum of the thermal driving force and the pump driving force where the pump pressure is much higher than the thermal driving pressure.

The pressure drop can be subdivided into two different groups: major and minor losses. Major losses involve the frictional pressure drops and minor losses involve the local pressure drops due to accessories in the cooling circuit (valves, junctions, etc.).

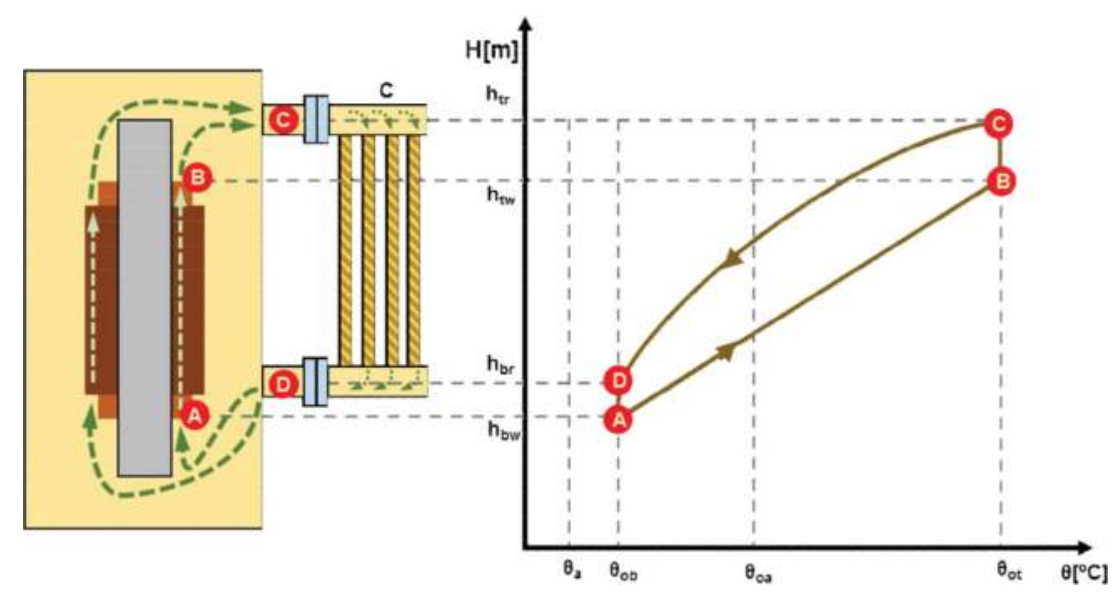

Figure 10. Oil loop of a transformer. 
The driving pressure on the loop must be equal to the total pressure drop. Energy conservation is also applied to the loop. The energy balance of the winding is presented in Eq. (17)

$$
p_{\gamma}=\rho \cdot c_{p} \cdot Q_{o} \cdot \Delta \theta_{o l}
$$

where $\mathrm{P} \gamma$ are the power losses in the winding $(\mathrm{W}), \mathrm{c}_{\mathrm{p}}$ is the specific heat of the oil at average oil temperature $(\mathrm{J} / \mathrm{kg} \cdot \mathrm{K}), \rho$ is the density at bottom-oil temperature $\left(\mathrm{kg} / \mathrm{m}^{3}\right)$ and $Q_{\mathrm{o}}$ is the volume oil flow $\left(\mathrm{m}^{3} / \mathrm{s}\right)$.

With these two equations, Eqs. (16) and (17), there are three variables that are volume oil flow $\mathrm{Q}_{\mathrm{o}}$, bottom-oil temperature $\mathrm{T}_{\mathrm{Ob}}$ and top-oil temperature $\mathrm{T}_{\mathrm{Ot}}$. Eq. (18) is added when considering the energy balance in the radiator

$$
k_{p} \cdot O \cdot\left(T_{0}(x)-T_{a}\right) d x=-\rho \cdot c_{p} \cdot Q_{0} \cdot d T_{0}(x)
$$

where $\mathrm{k}_{\mathrm{p}}$ is the total heat transfer coefficient $(\mathrm{HTC})\left(\mathrm{W} / \mathrm{m}^{2} \mathrm{~K}\right), \mathrm{O}$ is the circumference of the outer radiator cross section $(\mathrm{m}), \mathrm{T}_{\mathrm{O}}(\mathrm{x})$ is the oil temperature at position $\mathrm{x}(\mathrm{K})$ and $\mathrm{Q}_{\mathrm{O}}$ is the oil flow through the radiator $\left(\mathrm{m}^{3} / \mathrm{s}\right)$.

Assuming that the HTC does not change along the radiator in a significant way, the solution of Eq. (19) is

$$
T_{0}(x)=T_{a}+\left(T_{o t}-T_{a}\right) \cdot e^{-\frac{k_{p} \cdot 0}{\rho \cdot c_{p} \cdot Q_{0}} \cdot x}
$$

Then, integrating the cooling power in the radiator

$$
P=\int_{0}^{L_{R}}\left(T_{o}(x)-T_{a}\right) \cdot \mathrm{k}_{p} \cdot O \cdot d x
$$

result in the following equation:

$$
P=\rho \cdot c_{p} \cdot Q_{o} \cdot\left(T_{o t}-T_{a}\right) \cdot\left(1-e^{-\frac{k_{p} \cdot O \cdot L_{R}}{\rho \cdot c_{p} \cdot \ell_{o}}}\right)
$$

In this explanation, it is assumed that there is no heat exchange in the tank and there are no core losses. Consequently, from Eqs. (16), (17) and (21), two unknown temperatures and the oil flow can be determined [24].

\subsubsection{Detailed winding model}

THNM can be developed for predicting the temperature and oil flow distribution in a transformer winding in detail. For this type of modelling, bottom-oil temperature and oil flow rate have to be taken as inputs for the model. There is also the possibility to introduce a nonuniform power source in the active part of the winding. 
Applying a special discretization and following THNM principles, there are some assumptions that are taken in this kind of models: perfect thermal mixing is considered at junctions, fully developed flows are assumed in oil channels and exterior walls are considered as adiabatic.

The accuracy of these models has been tested and resulted acceptable. In order to increase this accuracy, many authors have tried to focus into two different parameters that come from correlations from datasets that are local pressure drop coefficients and convective heat transfer coefficient.

The knowledge of these two parameters is small in this application since there is not much experience to define them. However, some authors have defined these parameters based on datasets obtained from CFD results. Using CFD to accurately these two parameters has improved the performance of detailed THNMs. These kinds of models are known as CFD calibrated THNMs and integrate both main techniques of transformer thermal modelling.

THNM modelling predicts temperature distribution and velocity in the channels of the windings of the transformer. Hot-spot and top-oil temperatures can be estimated using THNM in a fast calculation, taking less than $5 \mathrm{~min}$ in a normal computer. These results show small deviations with respect to the obtained CFD models that are below $5 \%$ of deviation in hotspot and top-oil temperature.

\subsubsection{Detailed radiator model}

Other kinds of THNM modelling are the detailed radiator models. These models rely on the same principles than detailed winding models, but applied on the radiator part. Thermal modelling of radiators is complex, although radiators are mechanically simple, because of the following reasons: oil temperature varies in function of the height and per radiator panel, temperature variation is a function of the oil mass flow and the local heat flux, the local heat flux is dependent on the temperature difference between oil and ambient air and the local air velocity and the local air velocity are variable along the position in the radiator [25].

Especially, focus needs to be made on the air velocities distribution since there exist three possible configurations: air natural (AN), air forced (AF) with vertical air flow and air forced with horizontal flow. With AN configuration, the air flow through the panels is originated by buoyancy forces of the hot air. The buoyancy force will be in equilibrium with the pressure drop of the air flow through the panels.

In the case of $\mathrm{AF}$, air flow distribution generated from the fans has to be previously defined in order to obtain the heat transfer coefficient in the radiator plates. In order to better understand and model the air distribution over the radiator panels, CFD simulations have been carried out. Determining that the air coming from a fan spread in a conic way, an effective air velocity can be calculated based on the volumetric air capacity of the fan and the cone surface. With these assumptions, thermal modelling of the radiator can be made. 


\section{Summary}

In this chapter, different techniques for transformer thermal modelling have been introduced. The main goal of all of them is to predict the hot-spot temperature in the transformer windings with good accuracy. Due to the complex phenomena involved in transformer thermal modelling, the models have to be previously validated with experimental data. The first models are the dynamic models, which take into account different load factors to predict hot-spot and topoil temperatures over time. These models are useful to predict hot-spot temperatures in scenarios of emergency load. The steady-state models predict the temperature and velocity profiles in the windings of the transformer for a selected load rate. These models, CFD and THNM, are useful for design steps to predict the thermal behaviour of the transformer. CFD is a more accurate method, whereas THNM is faster and requires less computational resources. Both are used for design steps of transformer windings. Steady-state models are also used to test the cooling performance of alternative dielectric liquids, such as natural esters, in power transformers by comparing hot-spot temperature and pressure drop over the windings.

\section{Author details}

Agustín Santisteban*, Fernando Delgado, Alfredo Ortiz, Carlos J. Renedo and Felix Ortiz

*Address all correspondence to: santistebana@unican.es

ETSIIT University of Cantabria, Santander, Spain

\section{References}

[1] IEC Power transformers-Part 7: Loading guide for oil-immersed power transformers. IEC 60076-60077, 2011

[2] Susa D. Dynamic thermal modelling of power transformers. IEEE Transactions on Power Delivery, 0, 1. Jan 2005;20(1):197-204. ISSN: 0885-8977

[3] Susa D. Dynamic thermal modelling of power transformers: Further development-Part I. IEEE Transactions on Power Delivery, 10. Oct 2006;21(4):1961-1970. ISSN: 0885-8977

[4] Susa D. Dynamic thermal modelling of power transformers: Further development-Part II. IEEE Transactions on Power Delivery, 10. Oct 2006;21(4):1971-1980. ISSN: 0885-8977

[5] COMSOL Multiphysics. COMSOL Multiphysics Reference Manual. 2013. pp. 1-1262

[6] Torriano F, Chaaban M, Picher P. Numerical study of parameters affecting the temperature distribution in a disc-type transformer winding. Applied Thermal Engineering. 2010;30(14): 2034-2044. ISSN: 1359-4311. DOI 10.1016/j.applthermaleng.2010.05.004 
[7] Mufuta J, van den Bulck E. Modelling of the mixed convection in the windings of a disctype power transformer. Applied Thermal Engineering. 2000;20(5):417-437. ISSN: 13594311. DOI: 10.1016/S1359-4311(99)00034-4

[8] Wakil NE, Chereches N, Padet J. Numerical study of heat transfer and fluid flow in a power Transformer. International Journal of Thermal Sciences. Jun 1, 2006;45(6):615. ISSN: 1290-0729. DOI: 10.1016/j.ijthermalsci.2005.09.002

[9] Torriano F, Picher P, Chaaban M. Numerical investigation of 3D flow and thermal effects in a disc-type transformer winding. Applied Thermal Engineering. Jul, 2012;40:121-131. ISSN: 1359-4311. DOI: 10.1016/j.applthermaleng.2012.02.011

[10] Gastelurrutia J. et al. Numerical modelling of natural convection of oil inside distribution transformers. Applied Thermal Engineering. 2011;31:493-505. ISSN 1359-4311. DOI: 10.1016/ j.applthermaleng.2010.10.004

[11] Tsili MA, Amoiralis EI, Kladas AG, Souflaris AT. Power transformer thermal analysis by using an advanced coupled 3D heat transfer and fluid flow FEM model. International Journal of Thermal Sciences. Mar, 2012;53:188-201. CrossRef. ISSN: 1290-0729. DOI: 10.1016/j.ijthermalsci.2011.10.010

[12] Skillen A, Revell A, Iacovides H, Wu W. Numerical prediction of local hot-spot phenomena in transformer windings. Applied Thermal Engineering. Apr, 2012;36:96-105. ISSN: 13594311. DOI: 10.1016/j.applthermaleng.2011.11.054

[13] Yatsevsky VA. Hydrodynamics and heat transfer in cooling channels of oil-filled power transformers with multicoil windings. Applied Thermal Engineering. Feb, 2014;63(1):347353. CrossRef. ISSN: 1359-4311. DOI: 10.1016/j.applthermaleng.2013.10.055

[14] Torriano F. Numerical and experimental thermofluid investigation of different disc-type power transformer winding arrangements. The International Journal of Heat and Fluid Flow. 2018;69:62-72. ISSN: 0142-727X

[15] Park T. Numerical analysis of local hot-spot temperatures in transformer windings by using alternative dielectric fluids. Electrical Engineering. 2015;97(4):261-268. ISSN: 00039039

[16] Lecuna $\mathrm{R}$ et al. Thermal-fluid characterization of alternative liquids of power transformers: A numerical approach. IEEE Transactions on Dielectrics and Electrical Insulation. 2015;22(5):2522-2529. ISSN: 1070-9878. DOI: 10.1109/TDEI.2015.004793

[17] Santisteban A et al. Numerical analysis of the hot-spot temperature of a power transformer with alternative dielectric liquids. IEEE Transactions on Dielectrics and Electrical Insulation. 2017;24(5):3226-3235. ISSN: 1070-9878. DOI: 10.1109/TDEI.2017.006228

[18] Fernández $C$ et al. Thermal degradation assessment of kraft paper in power transformers insulated with natural esters. Applied Thermal Engineering. Jul 5, 2016;104:129-138. ISSN: 1359-4311. DOI: 10.1016/j.applthermaleng.2016.05.020 
[19] Kim M, Cho SM, Kim J. Prediction and evaluation of the cooling performance of radiators used in oil-filled power transformer applications with non-direct and direct-oil-forced flow. Experimental Thermal and Fluid Science. Jan 1, 2013;44:392. ISSN: 0894-1777. DOI: 10.1016/j.expthermflusci.2012.07.011

[20] Paramane SB. CFD study on thermal performance of radiators in a power transformer: Effect of blowing direction and offset of fans. IEEE Transactions on Power Delivery. 2014; 29(6):2596-2604. ISSN: 0885-8977

[21] Paramane SB. A coupled internal-external flow and conjugate heat transfer simulations and experiments on radiators of a transformer. Applied Thermal Engineering. 2016;103: 961-970. ISSN: 1359-4311

[22] Rodriguez GR. Numerical and experimental thermo-fluid dynamic analysis of a power transformer working in ONAN mode. Applied Thermal Engineering. 2017;112:1271-1280. ISSN: 1359-4311

[23] Garelli L. Reduced model for the thermo-fluid dynamic analysis of a power transformer radiator working in ONAF mode. Applied Thermal Engineering. 2017;124:855-864. ISSN: $1359-4311$

[24] Radakovic Z, Sorgic M. Basics of thermal-hydraulic model for thermal design of oil power transformers. IEEE Transactions on Power Delivery. 2010;25(2):790-802. ISSN: 0885-8977. DOI: 10.1109/TPWRD.2009.2033076

[25] van der Weken W, Paramane SB, Mertens M, Chandak V, Coddé J.. Increased efficiency of thermal calculations via the development of a full thermohydraulic radiator model. IEEE Transactions on Power Delivery. 2016;31(4):1473-1481. ISSN: 0885-8977. DOI: 10.1109/ TPWRD.2015.2501431 